\title{
Adaptive Sleep Mode Mechanism for 3G WiMax Networks
}

\author{
Arun M Bhaskaran ${ }^{1}$, Samundeeswari $\mathrm{P}^{2}$, Harikrishnan $\mathrm{M}^{3}$ \\ ${ }^{1}$ (Pondicherry University, Puducherry, India) \\ ${ }^{2}$ (Pondicherry University, Puducherry, India) \\ ${ }_{3}^{3}$ (Pondicherry University, Puducherry, India)
}

\begin{abstract}
The proliferation of wireless services and anytime anywhere communication providing always-on connectivity, mobile devices are getting smaller and smarter. Therefore, third-generation (3G) wireless standards IEEE 802.16e should provide very efficient energy conservation mechanisms to achieve longer battery life while providing enhanced user experience and quality of service (QoS). The emerging IEEE 802.16e standard provides a new sleep mode operation for Mobile Stations (MS) to address energy efficiency. In Sleep mode, the energy consumption of MS may be reduced by introducing a sleep cycle. However, a longer sleep cycle usually incurs higher response time. So there is a tradeoff between energy conservation and response time. Hence, we propose an Adaptive Sleep Mode Mechanism (ASMM) which adjusts an MS's sleep cycle in an adaptive manner based on online monitoring and estimation of the traffic condition. The goal is to minimize the response time of the MS while reducing the energy consumption. The effectiveness of the proposed method is verified by simulation results.
\end{abstract}

Keywords: Adaptive Sleep Mode Mechanism, IEEE 802.16e, Mobile Station, Response Time, Sleep Mode, Third Generation.

\section{Introduction}

WiMax based on IEEE 802.16e is a part of Third Generation (3G) communication technology. The sleep mode in IEEE 802.16e is designed to save Mobile Stations (MS) energy consumption when they are serviced with lightly-loaded traffic. Basically, an MS in the sleep mode wakes up to receive downlink data packets and/or exchange signaling messages with the Base Station (BS) during a listening interval, and powers down its transceiver during a sleep interval to reduce energy consumption. The interleaved listening and sleep interval repeat every sleep cycle as long as the MS is in the sleep mode.

Recently, there have been lots of studies conducted on sleep mode mechanism. Hwang [1] introduce an analytical model for the 802.16e sleep mode operation. However, their numerical analysis is based on an estimation obtained from an observation of simulation results, and hence, the numerical model may not be accurate. Xiao [2] analytically modeled the sleep-mode operation in IEEE 802.16e and evaluated the effects of minimum sleep interval, maximum sleep interval and packet arrival rate on networks performance metrics. Zhang[3] proposed an analytical model to evaluate energy management in IEEE 802.16e. In [4], Jun-Bae Seo investigated the queuing behavior of sleep-mode in terms of dropping probability and in terms of mean waiting time of packets in the queue of BS. In [5], the authors used a semi-Markov chain to derive packet delay and average energy consumption of sleeping, listening, and waking-up states. Junfeng [6] changed minimum sleep interval at low MAC SDU arrival rate to reduce energy consumption.

Jin [7] provided a model for the case that real time and non-real time packets are serviced simultaneously. The $802.16 \mathrm{e} / \mathrm{m}$ sleep mode has been well-studied in the previous works [8-10]. Zhu [11] presented a heuristic algorithm to determine the initial sleep window dynamically according to given traffic loads. Kim [12] introduced an efficient power management mechanism which takes into account the remaining power.

In Sleep mode, the energy consumption of MS may be reduced by introducing a sleep cycle. However, it is not considering the traffic arrival rate. In this paper Adaptive Sleep Mode Mechanism (ASMM) is proposed. Unlike in the legacy system, the length of the sleep interval is determined according to the traffic arrival rate of the serving Base Station (BS). Here the serving BS consider the inter arrival time into account to calculate the sleep interval so that the modified sleep mode mechanism consumes less energy compared with the original standard of sleep mode.

II. The Sleep Mode Operation in IEEE 802.16e

The sleep mode mechanism (SMM) is introduced in the IEEE 802.16e network is to reduce the energy consumed by a mobile station (MS). A mobile station has two modes awake mode and sleep mode. The MS in awake mode can send or receive data according to the Base Station (BS)'s scheduling. On the other hand, MS in the sleep mode can be absent from the serving BS during pre-negotiated intervals. Before switching to the sleep 
mode from the awake mode, the MS shall inform the BS using a sleep request message (MOB-SLP-REQ) and obtain its approval through a sleep response message (MOBSLP- RSP) from the BS. After receiving an MOBSLP-RSP message from the BS, the MS can enter the sleep mode.

The sleep mode involves two operational windows (i.e., time intervals), namely, sleep window and listening window, and an MS in the sleep mode basically switches between two windows. During a sleep window, an MS turns off most of its circuits in order to minimize the energy consumption, and hence cannot receive or transmit any message. If any packet destined to the MS in the sleep mode arrive at the BS during the sleep window of an MS, these packets are buffered so that they can be delivered to the MS when it is awake in the future. During a listening window, an MS synchronizes with its serving BS downlink and listens to a traffic indication message (MOB-TRF-IND), which indicates whether there is any buffered packet destined to the MS, to decide whether to stay awake to receive the pending packet or go back to sleep.

In order to reduce energy consumption, an MS alternates between awake-mode and sleep-mode during operation as it communicates with its BS. Before an MS goes into sleep mode, parameters for sleep-mode operation such as Tmin, Tmax, and listening interval, $L$, have to be set either by MSS or by its BS. With sleep response message (MOB-SLP-RSP) from the BS, the MSS enters sleep-mode. If the BS wants the MS to transit to awake-mode, it sends a positive indication message (MOB-TRF-IND) to the MSS during listening state. Then the MSS transits back to wake-mode after receiving a positive MOB-TRF-IND message. Fig. 1[1] shows the standard sleep mode in IEEE 802.16e.

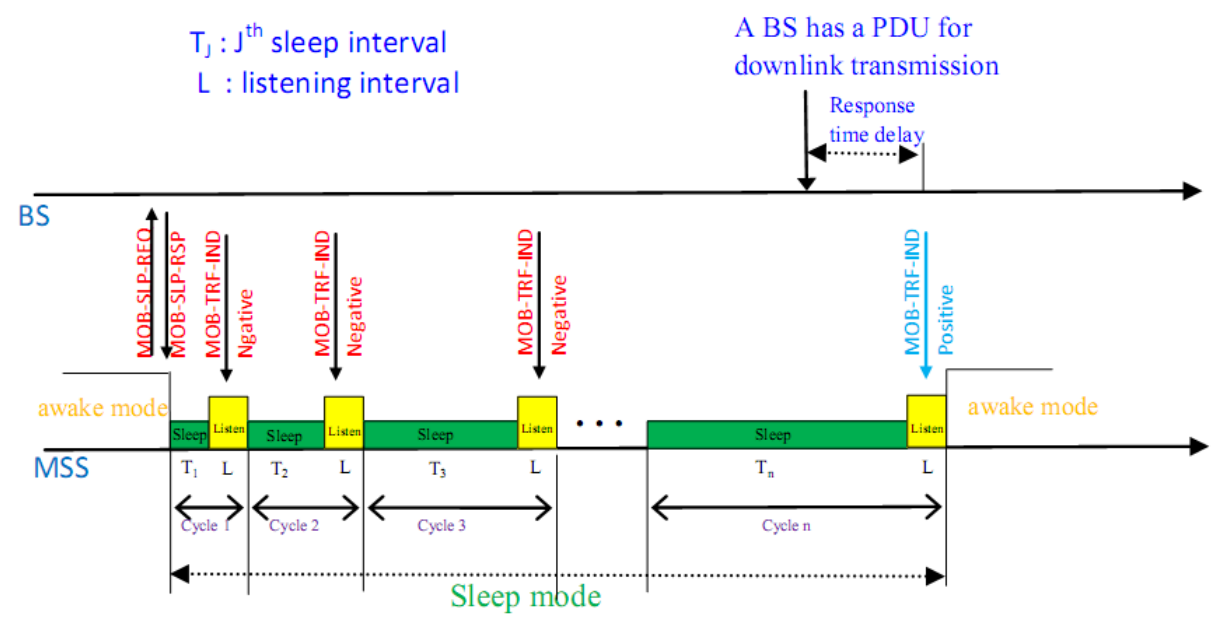

Fig. 1. IEEE 802.16e sleep mode

In 802.16e, the sleep intervals are defined as follows: At the first sleep interval, a minimum sleep interval Tmin is used. After the first sleep interval, an MS switches into a listening interval to wait for a MOBTRF-IND message. This message indicates addressed traffic that BS buffered for the MSS during previous sleep interval. The value of MOB-TRF-IND determines next MS's reaction. The negative MOB-TRF-IND message means that there is not any packet serving BS want to send. When an MSS receives a negative MOB-TRF-IND message, it goes to the next sleep interval whose duration is double from the preceding sleep interval. This process repeatedly continues until the sleep interval reaches to Tmax and then next sleep interval keeps unchanged. In $\mathrm{j}$-th listening interval, if serving BS sends the MSS a positive MOB-TRF-IND message, the MSS leaves sleep mode and wakes up to process packets. We call the duration of first sleep interval $\mathrm{T} 1=\mathrm{Tmin}$, then the duration of $\mathrm{j}$-th sleep interval is

$$
T_{j}=\min \left\{2^{j-1} T_{\min }, T_{\max }\right\}
$$

Let $\mathrm{n}$ and $\mathrm{D}$ denote the number of sleep intervals and the duration of a successive sequence of sleep intervals in sleep mode, respectively. L denote the listening interval. ES, EL denote the energy consumption units per unit time in the sleep interval and the listening interval, respectively. $\mathrm{R}$ denotes the frame response time which is defined as the delay a frame destined to an MS has to wait before it is delivered.

Assume that the arrival of frames destined to an MS follow a Poisson distribution with rate $\lambda$. It means, the inter arrival time is distributed according to an exponential law with parameter $1 / \lambda$. Let ej denote the event that there is at least one packet arrival during the monitor period $\mathrm{j}$. Note that in definition, listening intervals are also belong to the sleep mode.

$$
\operatorname{Pr}\left[e_{j}=\text { true }\right]=1-e^{-\lambda\left(T_{j}+L\right)}
$$

The term $\operatorname{Pr}[n=j]$ represents the probability of success in the exact $j$-th iteration, which is also the probability of failure in iteration 1 to $\mathrm{j}-1$ and success in the $\mathrm{j}$-th. The number of sleep cycles is an independent random variable. 


$$
\begin{aligned}
& \operatorname{Pr}(n=1)=\operatorname{Pr}\left(e_{1}=\text { true }\right)=1-e^{-\lambda\left(T_{j}+L\right)} \\
& \text { For } \mathrm{n} \geq 2, \operatorname{Pr}(n=j) \\
& =\operatorname{Pr}\left(e_{1}=\text { false } ; \ldots . ; e_{j-1}=\text { false } ; e_{j}=\text { true }\right) \\
& =\prod_{i=1}^{j-1} \operatorname{Pr}\left(e_{i}=\text { true }\right) \operatorname{Pr}\left(e_{j}=\text { true }\right) \\
& =e^{-\lambda \Sigma_{i=1}^{\tilde{j}-1}\left(T_{i}+L_{j}\right)}\left(1-e^{-\lambda\left(T_{j}+L_{L}\right)}\right. \\
& =\sum_{j=1}^{m} e^{-\lambda \Sigma_{\mathrm{i}=1}^{\mathrm{j}-1}\left(\Gamma_{\mathrm{i}}+L_{j}\right)}-\sum_{j=1}^{\mathrm{s}} e^{-\lambda \Sigma_{\mathrm{i}=1}^{\mathrm{j}}\left(\Gamma_{\mathrm{i}}+L_{j}\right)}
\end{aligned}
$$

From the equations (3) and (5), we can calculate the mean or average value of number of sleep intervals. Here $\mathrm{n}$ represents the number of sleep intervals and the expected value of $\mathrm{n}$ is represented by $\mathrm{E}[\mathrm{n}]$. Since, the value of $\mathrm{n}$ ranges from 0 to $\infty$, so the expected value of $\mathrm{n}$ is given by

$$
\begin{aligned}
& E[n]=\sum_{j=1}^{\infty} j \operatorname{Pr}(n=j) \\
& E[n]=\sum_{j=1}^{\mathrm{m}} e^{-\lambda \sum_{i=1}^{j-1}\left(\mathbb{T}_{\mathrm{i}}+L_{j}\right)}-\sum_{j=1}^{\infty} e^{-\lambda \sum_{i=1}^{j}\left(\mathbb{T}_{\mathrm{i}}+\mathrm{L}_{j}\right)}
\end{aligned}
$$

Each sleep cycle has length of Tj+L. Therefore, the expected duration of a sequence of sleep mode cycles is calculated by:

$$
\begin{aligned}
& E[D]=\sum_{j=1}^{m} \operatorname{Pr}(n=j)(j-\text { th cycle duration }) \\
& =\sum_{j=1}^{\infty} \operatorname{Pr}(n=j) \sum_{k=1}^{j}\left(T_{k+} L\right) \\
& =\sum_{j=1}^{\mathrm{s}} e^{-\lambda \Sigma_{\mathrm{i}=1}^{\mathrm{j}} \mathrm{i}\left(\mathbb{I}_{\mathrm{i}}+\mathrm{L}_{j}\right)}-\sum_{j=1}^{\mathrm{s}} e^{-\lambda \Sigma_{\mathrm{i}=1}^{\mathrm{j}}\left(\mathrm{T}_{\mathrm{i}}+\mathrm{L}\right)} \sum_{k=1}^{j}\left(T_{k+} L\right)
\end{aligned}
$$

The energy consumption for $\mathrm{j}$-th cycle is $E_{j}=\sum_{k=1}^{j}\left(T_{k} E_{g}+L E_{L}\right)$. Hence, the expected energy consumption of a sequence of sleep mode cycles is,

$$
\begin{aligned}
E[\text { Energy] } & =\sum_{j=1}^{\mathrm{m}} \operatorname{Pr}(n=j) E_{j} \\
& =\sum_{j=1}^{\mathrm{m}} \operatorname{Pr}(n=j) \sum_{k=1}^{j}\left(T_{k} E_{g}+L E_{L}\right) \\
& =\sum_{j=1}^{\mathrm{m}} e^{-\lambda \sum_{i=1}^{\mathrm{j}-1}\left(T_{\mathrm{i}}+\mathrm{L}\right)} \sum_{k=1}^{j}\left(T_{k} E_{g}+L E_{L}\right)-\sum_{j=1}^{\mathrm{m}} e^{-\lambda \sum_{i=1}^{\mathrm{j}}\left(T_{\mathrm{i}}+L\right)} \sum_{k=1}^{j}\left(T_{k} E_{g}+L E_{L}\right)
\end{aligned}
$$

It's assumed that the frame causing escape from the sequence of sleep mode cycles will arrive at any moment during the last cycle with uniform probability. The length of $\mathrm{j}$-th cycle is $\left(T_{j}+L\right)$. The expected frame response time is defined as:

$$
\begin{aligned}
& E[R]=\sum_{j=1}^{m} \operatorname{Pr}(n=j) \sum_{k=1}^{j}\left(T_{j}+L\right) / 2 \\
& =\frac{1}{2} \sum_{j=1}^{\infty} e^{-\lambda \sum_{i=1}^{j-1}\left(T_{i}+L\right)}\left(T_{j}+L\right)-\frac{1}{2} \sum_{j=1}^{\infty} e^{-\lambda \sum_{i=1}^{j}\left(T_{i}+L\right)}\left(T_{j}+L\right)
\end{aligned}
$$

\section{The Proposed Adaptive Sleep Mode Mechanism}

In this section, we present our proposed adaptive sleep mode mechanism, called ASMM. ASMM is executed at the BS. It (1) monitors downlink packets for each MS and estimates the packet arrival rate online, based on which (2) it adjusts the sleep cycle for the MS and uses the sleep request message (MOB-SLP-REQ) to notify the station of the new sleep cycle length. Once the MS receives MOB-SLP-REQ, it starts the next sleep cycle following the BS's instruction.

Based on the above analysis, we can see that these parameters Tmin, Tmax are main factors affecting on the energy consumption and frame response time (delay). In general, each pair of (Tmin, Tmax) gives different results of energy consumption and response delay. So, if the value of Tmin and Tmax are limited in a given range, we certainly choose a pair (Tmin, Tmax) which causes energy consumption and response delay will be minimum correspond to a certain $\lambda$. The algorithm to choose (Tmin_ $\lambda$, Tmax $\_\lambda$ ) is below:

Step 1: Initialise $\mathrm{j}=0, \mathrm{Tmin}=1, \mathrm{Tmax}=1024$

Step 2: $\quad$ Calculate tmin $=2^{\mathrm{j}} \mathrm{Tmin}$

Step 3: $\quad$ Calculate tmax $=2^{\mathrm{j}}$ tmin

Step 4: $\quad$ If $(\operatorname{tmax}<\mathrm{Tmax})$

Else

Go to step 5

Go to step 6

Step 5: $\quad$ If(Energy $(\operatorname{tmin}, \operatorname{tmax}, \lambda)<\min \_$energy $\&$ Delay $(\operatorname{tmin}, \operatorname{tmax}, \lambda)<\min \_$delay $)$

Tmin $\_\lambda=\operatorname{tmin}, T \operatorname{Tmax} \_\lambda=\operatorname{tmax}$

min_energy $=$ Energy $(\operatorname{tmin}, \operatorname{tmax}, \lambda)$

$\min \_$delay=Delay $(\operatorname{tmin}, \operatorname{tmax}, \lambda)$

Else

Go to step 6 
Step 6: $\quad \mathrm{j}=\mathrm{j}+1$ and go back to step 3

Step 7: $\quad$ If $($ tmin $<$ Tmax $)$

Else

Go to step 8

Go to step 9

Step 8: $\quad \mathrm{j}=\mathrm{j}+1$ and go back to step 2

Step 9: $\quad$ Energy=min_energy

Delay=min_delay

By scanning all possible cases of (Tmin, Tmax), we can choose the best case which has minimum energy consumption and minimum delay for given $\lambda$. To compare new mechanism, we chosen Tmin $=1$, Tmax $=1024, \mathrm{~L}=1$. Sleep energy consumption (ES) and listening energy consumption (EL) are $E S=1, E L=30$. We consider $\lambda$ in range of $[0.001 ; 0.2]$. For the each packet arrival rate $(\lambda)$ we will take one Tmin value and consider the different Tmax values. By using those values we will calculate the energy consumption for the particular packet arrival rate.

For a particular packet arrival rate we will calculate the energy consumption for all the possible values of Tmin and Tmax. This calculation will be repeated for the different packet arrival rates. From these values we will find out the best pair of Tmin and Tmax values which will provide very less energy consumption and response delay. This Tmin and Tmax values are used as the minimum sleep interval and maximum sleep interval for a particular packet arrival rate. By using the Tmin and Tmax values we will calculate the energy consumption, expected number of sleep intervals and the response delay. Here unlike in the legacy system the sleep cycle is dynamically changing according to the packet arrival rate.

(i) Energy Saving

The energy saved by the proposed Adaptive Sleep Mode Mechanism compared to the Sleep Mode Mechanism can be calculated by the following formula:

$$
\text { Energy Saving }(100 \%)=\frac{\text { Energy }_{\text {PSM }}-\text { Energy }_{M E S}}{\text { Energy }_{F M}} X 100 \%
$$

The effectiveness of the proposed Adaptive Sleep Mode Mechanism can be determined by how many percentage of energy is saved.

\section{Results and Discussion}

In this section, we compare the simulation results of the standard Sleep Mode Mechanism (SMM) with proposed Adaptive Sleep Mode Mechanism (ASMM). The SMM and the proposed ASMM are implemented using Matlab 2012b. The expected values of sleep intervals, duration, response delay and energy consumption are simulated for the existing and the proposed methods and the results are compared. The proposed algorithm ensures less energy consumption and response delay compared to the present method. The parameters used in simulation are: $\mathrm{Tmin}=1, \mathrm{Tmax}=1024, \mathrm{~L}=1, \mathrm{ES}=1, \mathrm{EL}=30$.

In the proposed Adaptive Sleep Mode Mechanism (ASMM) the sleep interval is dynamically changed according to the packet arrival rate. Fig. 2 shows the expected value of number of sleep intervals of Sleep Mode Mechanism and Adaptive Sleep Mode Mechanism. In the ASMM expected value of number of sleep intervals for a particular packet arrival rate is more compared to the existing method.

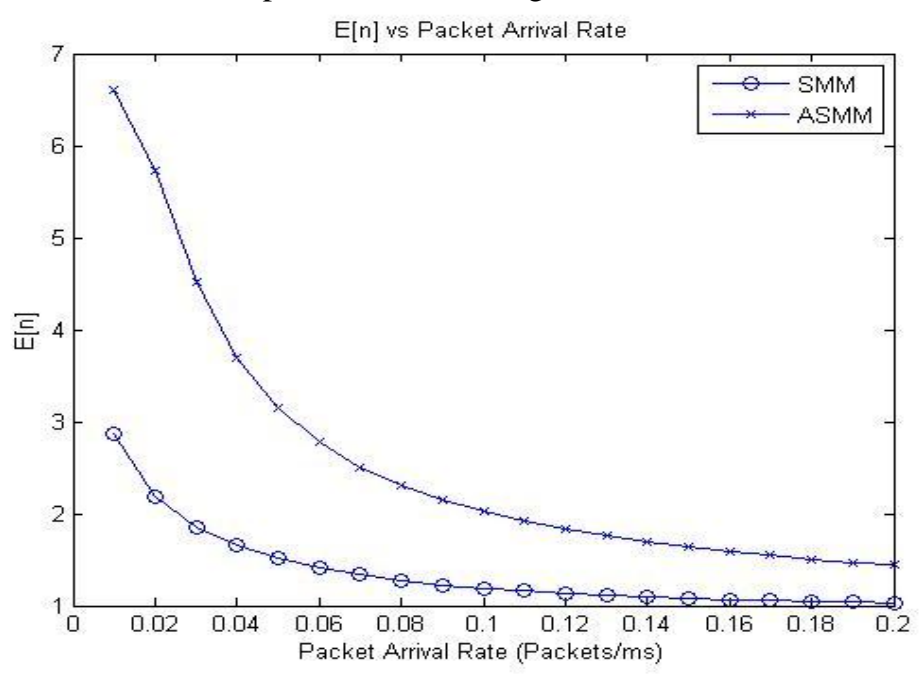

Fig. 2 expected value of number of sleep intervals 
Fig. 3 shows the comparison of expected value of successive sequence of sleep duration of Sleep Mode Mechanism and Adaptive Sleep Mode Mechanism. Here, the sleep duration of proposed method is less than the existing method. The sleep duration is decreased because number of sleep intervals is increased in the proposed method. When the packet arrival rate is low, the sleep duration and number of sleep intervals will be high. And whenever the packet arrival rate is high the number of sleep intervals and sleep duration will be less.

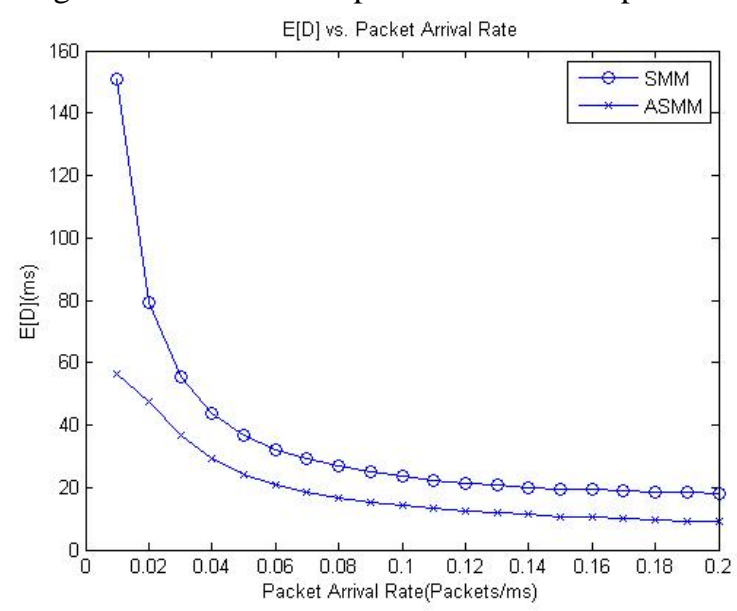

Fig. 3 expected value of sleep duration

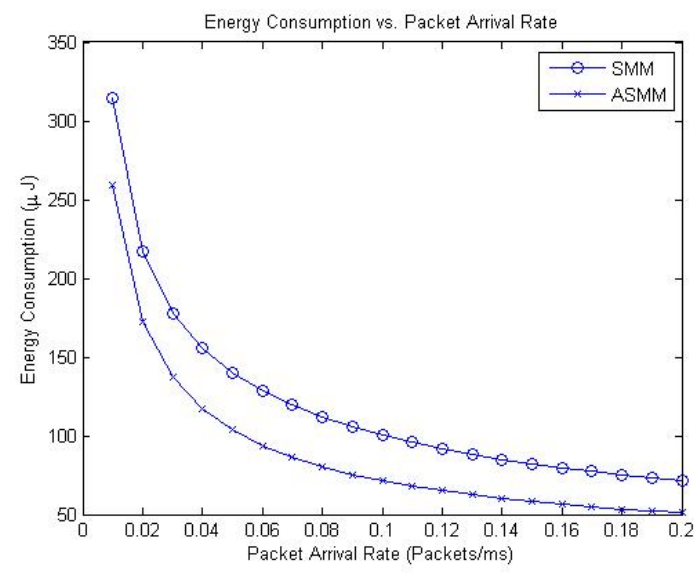

Fig. 4 energy consumption

Fig. 4 shows the comparison of energy consumption of Sleep Mode Mechanism and Adaptive Sleep Mode Mechanism. It shows that the proposed mechanism having less energy consumption compared to the existing Sleep Mode Mechanism. In the proposed ASMM sleep mode operation the sleep cycle dynamically changes according to the packet arrival rate. Here, for each $\lambda$ we are finding out the minimum and maximum value of sleep interval which will give the lesser energy consumption. So the energy consumption of the proposed method will give better energy conservation.

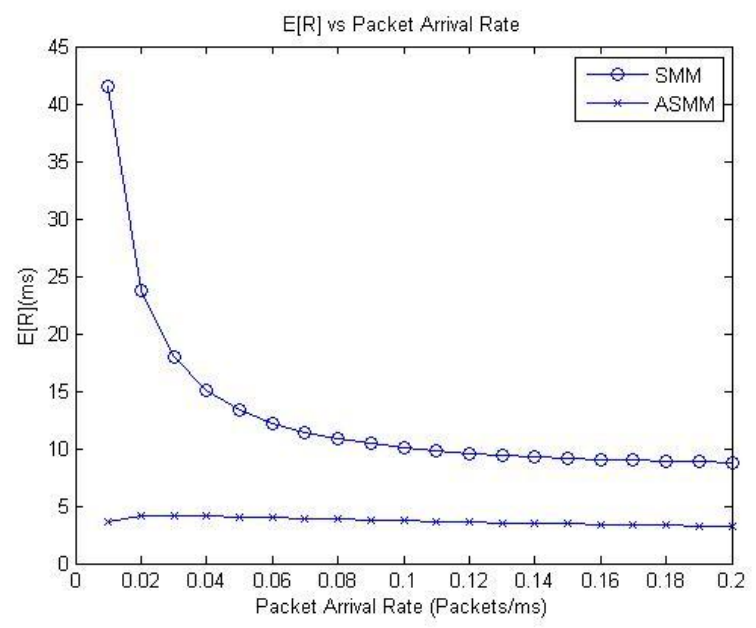

Fig. 5 response delay 
Fig. 5 shows the comparison of frame response delay versus packet arrival rate of the Sleep Mode Mechanism and Adaptive Sleep Mode Mechanism. Here the proposed mechanism having reduced frame response time than the existing SMM method.

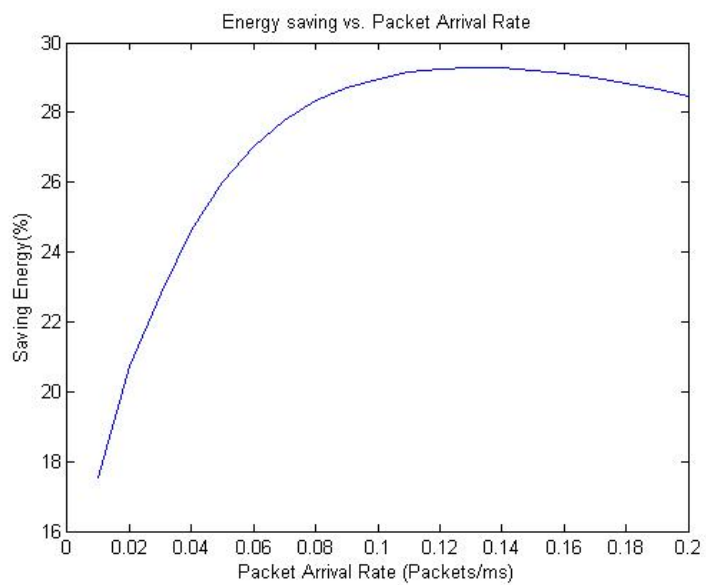

Fig. 6 energy saving

Fig. 6 shows the energy saving obtained by the proposed MSMM mechanism. It shows that the ASMM saved more than $18 \%$ of energy compared with the Sleep Mode Mechanism.

\section{Conclusion}

In this paper, we introduced Adaptive Sleep Mode Mechanism to minimize the energy consumption and response delay. The Adaptive Sleep Mode Mechanism for IEEE 802.16e dynamically changes the sleep interval based on traffic rate of serving Base Station. The simulation results confirm that the proposed Adaptive Sleep Mode Mechanism having less response delay and the new mechanism saved more than $18 \%$ energy compared with the existing Sleep Mode Mechanism.

\section{References}

[1] E. Hwang, K.J. Kim, J.J. Son, B.D. Choi, "The power-save mechanism with periodic traffic indications in the IEEE 802.16e/m," IEEE Trans. Veh. Tech., vol 46 ,pp 2176-2185,January 2010.

[2] Yang Xiao, "Performance analysis of an energy saving mechanism in the IEEE 802.16e wireless MAN," CCNC 2006. 2006 3rd IEEE Consumer Communications and Networking Conference, 2006., Las Vegas, NV, USA: , pp. 406-410.

[3] Yan Zhang, Yang Xiao, and V.C.M. Leung, "Energy Management Analysis and Enhancement in IEEE 802.16e WirelessMAN," IEEE Transactions on Vehicular Technology, vol. 58, Sep. 2009, pp. 3738-3752

[4] Jun-Bae Seo, Seung-Que Lee, and Nam-Hoon Park, "Performance Analysis of Sleep Mode Operation in IEEE 802.16e," IEEE Vehicular Technology Conference, vol.2, pp. 1169 - 1173, Sept. 2004

[5] Junfeng XIAO, Shihong ZOU, Biao REN, Shiduan CHENG, "An Enhanced Energy Saving Algorithm in IEEE 802.16e", IEEE Global Communication, Dec. 2006

[6] S. Zhu and T. Wang, "Enhanced power efficient sleep mode operation for IEEE 802.16e based WiMAX," 2007 IEEE Mobile WiMAX Symposium, Orlando, FL, USA: 2007, pp. 43-47.

[7] S. Jin, M. Choi, S. Choi, "Performance analysis of IEEE 802.16m sleep mode for heterogeneous traffic," IEEE Commun. Lett., May 2010.

[8] Yu-Chee Tseng, Jen-Jee Chen and Yen-Chih Yang, " Managing Power Saving Classes in IEEE 802.16 Wireless MANs: A Foldand-Demultiplex Method," IEEE Trans. on Mobile Computing, Vol. 10, No. 9, pp 1237-1247, September 2011.

[9] Fuad M. Abinader Jr., Vicente A. de Sousa Jr., N’ibia S. Bezerra, Gino Lozada, Pekko Orava, "Performance of Power Saving Modes in IEEE 802.16e System," IEEE Transactions on Mobile Computing, vol. 8, no. 4, pp 198-206, April 2009.

[10] Alexey Anisimov, Sergey Andreev, Andrey Turlikov, "IEEE 802.16m Energy-Efficient Sleep Mode Operation Analysis with Mean Delay Restriction," IEEE Trans. Veh. Tech., vol 39,pp 1141-1151,January 2010.

[11] S. Zhu and T. Wang, "Enhanced power efficient sleep mode operation for ieee 802.16e based wimax," IEEE Mobile WiMAX Symposium, 2007

[12] M.-G. Kim, J. Choi, B. Jung, and M. Kang, "Adaptive power management mechanism considering remaining energy in IEEE 802.16e," IEICE Trans. Commun., vol. E90-B, no. 9, pp. 2621-2624, Sept. 2007. 